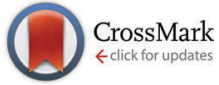

Cite this: Phys. Chem. Chem. Phys. 2014, 16, 20492

Received 9th July 2014,

Accepted 7th August 2014

DOI: 10.1039/c4cp03009e

www.rsc.org/pccp

\title{
Shining new light on the multifaceted dissociative photoionisation dynamics of $\mathrm{CCl}_{4}$
}

\begin{abstract}
Jonelle Harvey,$\dagger^{a}$ Richard P. Tuckett $\star^{a}$ and Andras Bodi ${ }^{b}$
Internal energy selected carbon tetrachloride cations have been prepared by imaging photoelectron photoion coincidence (iPEPICO) spectroscopy using synchrotron vacuum ultraviolet radiation. The threshold photoelectron spectrum shows a newly observed vibrational progression corresponding to the $\nu_{2}(e)$ scissors mode of $\mathrm{CCl}_{4}^{+}$in the third, $\tilde{\mathrm{B}}{ }^{2} \mathrm{E}$ band. Ab initio results on the first four doublet and lowest-lying quartet electronic states along the $\mathrm{Cl}_{3} \mathrm{C}^{+}-\mathrm{Cl}$ dissociation coordinate show the $\tilde{\mathrm{B}}$ state to be strongly bound, and support its relative longevity. The $\tilde{X}^{2} T_{1}$ and $\tilde{A}^{2} T_{2}$ cationic states, on the other hand, are barely bound and dissociate promptly. The $\tilde{\mathrm{C}}^{2} \mathrm{~T}_{2}$ state may intersystem cross to the quartet a state, which dissociates to a triplet state of the $\mathrm{CCl}_{3}{ }^{+}$fragment ion. This path is unique among analogous $\mathrm{MX}_{4}{ }^{+}(\mathrm{M}=\mathrm{C}, \mathrm{Si}, \mathrm{Ge} ; \mathrm{X}=\mathrm{F}$, $\mathrm{Cl}, \mathrm{Br}$ ) systems, among which several have been shown to have long-lived $\tilde{\mathrm{C}}$ states, which decay by fluorescence. The breakdown diagram, recorded here for the first time for the complete valence photoionisation energy range of $\mathrm{CCl}_{4}$, is interpreted in the context of literature based and CBS-QB3, G4, and W1U computed dissociative photoionisation energies. $\mathrm{No} \mathrm{Cl}_{2}$-loss channel is observed in association with the $\mathrm{CCl}_{2}{ }^{+}$or $\mathrm{CCl}^{+}$ fragments below the 2 or $3 \mathrm{Cl}$-loss reaction energies, and $\mathrm{Cl}_{2}$ loss is unlikely to be a major channel above them. The breakdown diagram is modelled based on the calculated dissociative photoionisation onsets and assuming a statistical redistribution of the excess energy. The model indicates that dissociation is not impulsive at higher energies, and confirms that the $\tilde{C}^{2} \mathrm{~T}_{2}$ state of $\mathrm{CCl}_{4}{ }^{+}$forms triplet-state $\mathrm{CCl}_{3}{ }^{+}$fragments with some of the excess energy trapped as electronic excitation energy in $\mathrm{CCl}_{3}{ }^{+}$.
\end{abstract}

\section{Introduction}

A chemical reaction can take place if there is more kinetic energy in one degree of freedom, the reaction coordinate, than the activation energy. Therefore, unveiling internal energy flows and their effect on the reactive flux are central to understanding chemical dynamics. Among different electronically excited states, the reactive flux is guided by internal conversion and intersystem crossings. The non-ergodic nature of the former has been the subject of a recent minireview by Sølling et al. ${ }^{1}$ Among other phenomena, they discuss the localization of the internal energy in one degree of freedom, e.g., a reactive mode, as dictated by internal conversion as well as the induction of internal conversion by a certain molecular motion. In the neutral, such processes can be followed directly by femtosecond pump-probe spectroscopy. ${ }^{2,3}$ In the ionic manifold, internal energy selected parent ions can be prepared using threshold

\footnotetext{
${ }^{a}$ School of Chemistry, University of Birmingham, Edgbaston, Birmingham B15 2TT, UK. E-mail: r.p.tuckett@bham.ac.uk

${ }^{b}$ Molecular Dynamics Group, Swiss Light Source, Paul Scherrer Institut,

Villigen 5232, Switzerland. E-mail: andras.boedi@psi.ch

$\dagger$ Current address: The Royal Society of Chemistry, Thomas Graham House, Science Park, Milton Road, Cambridge CB4 OWF, UK.
}

photoionisation and tunable vacuum ultraviolet (VUV) light. ${ }^{4}$ Their unimolecular dissociation dynamics can then be studied by imaging photoelectron photoion coincidence (iPEPICO) spectroscopy at the VUV beamline of the Swiss Light Source. ${ }^{5,6}$ Dissociative photoionisation thresholds and measured dissociation rates in the $10^{3}-10^{7} \mathrm{~s}^{-1}$ range can routinely be interpreted by statistical theory, ${ }^{7}$ to derive thermochemical data with accuracy sometimes below $1 \mathrm{~kJ} \mathrm{~mol}^{-1}$. ${ }^{8,9}$ Statistical theory assumes that the complete phase space is explored by the system, and the dissociation rate essentially corresponds to the probability that sufficient kinetic energy is present in the reaction coordinate.

Statistical processes pre-suppose the presence of a bound state of the parent cation. As, at any internal energy, the phase space volume of the ground state is much larger than that of the electronically excited states, dissociation normally takes place on this potential energy surface, following internal conversion from higher-lying states. Thus, the assumption of statistical behaviour relies on strong coupling between different degrees of freedom. Anharmonicity is the driving force of intramolecular vibrational energy redistribution (IVR), which explains why non-statistical behaviour is observed for low-barrier isomerization reactions of large molecules in which the internal energy per oscillator, and thus, the anharmonicity, are small at the activation energy. ${ }^{10,11}$ Electronic degrees of freedom are coupled by conical intersections. 
The large density of states in cations allows for the observation of Franck-Condon prohibited non-resonant transitions in threshold photoionisation, and ensures that the electronic excitation energy is also available for dissociation. ${ }^{12}$ In recent years, we made use of the tunability and energy resolution of the iPEPICO endstation and revisited several halogenated systems to study their dissociative photoionisation properties. In agreement with previous observations, we have found a number of non-statistical processes. As best shown in the case of the fluoroethene cations, $\mathrm{C}_{2} \mathrm{H}_{4-n} \mathrm{~F}_{n}^{+} \quad(n=1-4)$, new experimental and theoretical approaches have yielded a deeper understanding of the underlying reaction mechanism than was possible before. ${ }^{13,14}$ These cations dissociate statistically in the low-energy region along several parallel dissociation channels. In the mid- to high-valence ionisation energy region, they lose $\mathrm{F}$ atoms in a non-statistical process. In mono-, di-, and trifluoroethene, it was shown that $\tilde{\mathrm{C}}$-state cations can undergo internal conversion to the $\tilde{\mathrm{B}}$ state, either on a bound or a repulsive domain along the $\mathrm{C}-\mathrm{F}$ bond stretch coordinate. Should the latter take place, the product $\tilde{\mathrm{B}}$ state cation suffers non-statistical F-loss promptly. Long-lived $\tilde{\mathrm{B}}$-state ions, on the other hand, can redistribute their internal energy, and dissociate statistically. $\mathrm{In}_{2} \mathrm{~F}_{4}{ }^{+}$, the electronically excited $\tilde{\mathrm{A}}$ state is disconnected from the ground state and only correlates with ground state $\mathrm{C}_{2} \mathrm{~F}_{3}{ }^{+}+\mathrm{F}$ products. Hence, the $\tilde{\mathrm{A}}$ state establishes a second dissociation regime, which is disconnected from the low-energy regime solely because of an absence of electronic coupling.

In this paper, we report on the dissociative photoionisation dynamics of carbon tetrachloride. We show that, while the dissociation is impulsive close to the onset of ionisation, it takes on a statistical character as coupling to the repulsive ground state of the parent cation gets weaker with higher electronic excitation.

Another non-statistical decay process should now be mentioned, namely fluorescence. ${ }^{15}$ The cations of analogous halogenated compounds are known to fluoresce, ${ }^{16}$ but it is often difficult to identify fluorescence competing with fragmentation processes. ${ }^{17}$ If dissociation is energetically prohibited, the only decay process for excited electronic states in the gas phase is fluorescence, and such ions will indeed fluoresce. Radiative decay decreases the available energy for fragmentation processes. As will be discussed later, unlike in analogous $\mathrm{MX}_{4}{ }^{+}$ions, fluorescence processes have not been observed in $\mathrm{CCl}_{4}{ }^{+}$, and fluorescence cannot compete effectively with dissociative photoionisation whenever the latter is energetically allowed.

Several of the carbon group tetrahalogenides of $\mathrm{MX}_{4}(\mathrm{M}=\mathrm{C}, \mathrm{Si}$, $\mathrm{Ge} ; \mathrm{X}=\mathrm{F}, \mathrm{Cl}, \mathrm{Br}, \mathrm{I}$ ) are unstable with respect to photoionisation, i.e. the ground electronic state of $\mathrm{MX}_{4}^{+}$in the Franck-Condon window is unbound, but the cations also possess bound excited electronic states. Carbon tetrachloride, $\mathrm{CCl}_{4}$ dissociatively photoionises into daughter ions $\mathrm{CCl}_{3}{ }^{+}, \mathrm{CCl}_{2}{ }^{+}, \mathrm{CCl}^{+}$in the $11-30 \mathrm{eV}$ photon energy range, and a very weak $\mathrm{CCl}_{4}{ }^{+}$peak can only be observed under special circumstances. ${ }^{18,19}$ Similar to $\mathrm{CCl}_{4}{ }^{+}$, the lower electronic states of $\mathrm{CF}_{4}{ }^{+}$were also proposed to be repulsive, resulting in a significant force towards $\mathrm{C}-\mathrm{F}$ bond length increase in the Franck-Condon allowed photoionisation energy range, and giving rise to an impulsive dissociation to form $\mathrm{CF}_{3}{ }^{+}+\mathrm{F}+\mathrm{e}^{-20}$
Kinugawa et al. published photoion and photoelectron angular distribution data on VUV ionisation of $\mathrm{CF}_{4}$ and $\mathrm{CCl}_{4}$, and, based on the observed correlation, suggested that electron and nuclear motion may take place on similar time-scales. ${ }^{21}$ As seen from our work on fluoroethenes and from the amount of new thermochemical information derived from the breakdown diagrams of halogenated methanes, ${ }^{22}$ the higher internal energy resolution of the iPEPICO experiment can offer further insights into dissociative photoionisation.

In threshold photoion photoelectron coincidence (TPEPICO) spectroscopy, virtually zero kinetic energy electrons are detected and used as the start signal for ion time-of-flight (TOF) analysis. Method developments in the last decade included the application of velocity map imaging for high collection efficiency, ${ }^{23}$ slow extraction fields for high residence times to measure dissociation rates, ${ }^{4}$ the use of synchrotron radiation ${ }^{6,24,25}$ together with fast position-sensitive detectors and triggerless data acquisition ${ }^{26}$ setups, and, most recently, double imaging experiments. ${ }^{27,28}$ In addition to yielding more accurate onsets and broadening the spectrum of possible samples, these improvements have led to a refined understanding of threshold photoionisation, ${ }^{12}$ as well as detailed models for the non-statistical aspects of the dissociative photoionisation of fluoroethenes, ${ }^{13,14}$ halogenated tin compounds ${ }^{29}$ and methanol. ${ }^{30}$ Consequently, it seemed fitting to re-visit the impulsive halogen loss from $\mathrm{CCl}_{4}^{+}$, a system known to exhibit non-statistical behaviour, in more detail using the iPEPICO experiment. ${ }^{31}$

\section{Experimental methods}

Experiments were performed using the iPEPICO endstation at the VUV beamline of the Swiss Light Source (SLS) at the Paul Scherrer Institut in Villigen, Switzerland. The setup has been described in detail in several publications and only an overview is given here. ${ }^{5,6,26}$ Carbon tetrachloride vapour was introduced effusively into the ionisation chamber of the iPEPICO endstation at room temperature, where it was ionised by the incident synchrotron radiation. Typical operating pressures within the chamber were $7-9 \times 10^{-7}$ mbar. The synchrotron radiation is dispersed by a grazing-incidence monochromator with laminar 600 and $1200 \mathrm{~mm}^{-1}$ gratings for photon energies below and above $15 \mathrm{eV}$, respectively. The photon energy at a resolving power, $E / \Delta E$, of $c a$. 5000 was calibrated against the $11 \mathrm{~s}^{\prime}$ to $14 \mathrm{~s}^{\prime}$ argon autoionisation lines in both first and second order. In addition to the laminar gratings, higher harmonic radiation is further suppressed by a compact differentially pumped rare-gas filter operating at 10 mbar. Pure neon was used in the gas filter, applicable to the 11-21 eV photon energy range used herein.

Photoelectrons are extracted with a continuous field and are velocity map imaged onto a DLD40 Roentdek position sensitive delay-line detector. Threshold electrons are focussed into a small centre spot on the detector with a kinetic energy resolution better than $1 \mathrm{meV}$. Photoions are extracted in the opposite direction by the same, constant $120 \mathrm{~V} \mathrm{~cm}^{-1}$ field in the first $5 \mathrm{~cm}$ long acceleration region. Afterwards, they are accelerated further to 
achieve space focussing, pass through a $50 \mathrm{~cm}$ long field-free drift region, then are detected by a Jordan TOF C-726 microchannel plate detector. Photoelectrons are position and time stamped, and serve as the start signal for the ion time-of-flight analysis.

Some energetic (or hot) electrons are produced with negligible off-axis momentum, and are also focussed into the centre spot, contaminating the true threshold signal. The hot electron contamination is accounted for by a subtraction process, ${ }^{32,33}$ whereby a small ring area around the centre is assumed to represent the hot electron background in the centre, and the time-of-flight mass spectrum in coincidence with ring electrons is multiplied with a factor corresponding to the centre-to-ring area ratio and subtracted from the mass spectrum in coincidence with the central electrons. Photoion mass selected threshold photoelectron spectra (ms-TPES) indicate the threshold photoionisation yields of different ions as a function of photon energy. The fractional ion abundances can be plotted as a function of photon energy in the breakdown diagram, whereas all electrons can be used to plot the threshold photoelectron spectrum of the sample.

\section{Computational methods}

Calculations were carried out using the Gaussian $09^{34}$ as well as the QChem 4.0.1 programs. ${ }^{35}$ The former was used to determine the reaction energies of different dissociative photoionisation channels of $\mathrm{CCl}_{4}$ using the CBS-QB3, G4 and W1U composite methods. DFT geometry optimizations at the B3LYP/6-311+G(d) level mapped the potential energy surface. In these geometry optimizations, two bond lengths were scanned in $C_{3 \mathrm{v}}$ symmetry, namely a single $\mathrm{C}-\mathrm{Cl}$ bond length for the leaving chlorine atom, and a $\mathrm{C}-\mathrm{Cl}$ bond length in the remaining $\mathrm{CCl}_{3}{ }^{+}$fragment. The reaction coordinate was then defined as a minimum energy path on this two-dimensional surface.

EOM-IP-CCSD (equation-of-motion coupled-cluster singles and doubles for ionisation potentials) calculations ${ }^{36}$ were carried out with the cc-pVTZ basis set to obtain the energies of the doublet ${ }^{2} \tilde{\mathrm{X}}$, ${ }^{2} \tilde{\mathrm{A}},{ }^{2} \tilde{\mathrm{B}}$, and ${ }^{2} \tilde{\mathrm{C}}$ cation states along the $\mathrm{C}-\mathrm{Cl}$ dissociation coordinate in $\mathrm{CCl}_{4}{ }^{+}$. Second order Møller-Plesset perturbation theory (MP2) was also used along the same path to calculate the energy difference between the doublet ${ }^{2} \tilde{\mathrm{X}}$ and the quartet ${ }^{4}$ ã states, which was then added to the ground state reaction energy curve to obtain a path to Cl-loss on the lowest-lying quartet surface.

\section{Results and discussion}

\section{Threshold photoelectron spectrum}

The photoelectron spectrum of $\mathrm{CCl}_{4}$ was previously measured by von Niessen et al., ${ }^{37}$ and our threshold photoelectron spectrum is shown in Fig. 1. The photoelectron peak for the $\tilde{X}^{2} T_{1}$ state is broad and structureless, as is expected for a vertical transition into an unbound domain of the cation potential energy surface. The spin-orbit splitting of the next, $\tilde{\mathrm{A}}^{2} \mathrm{~T}_{2}$ peak, $c a .0 .22 \mathrm{eV}$, is, however, readily observable. The higher degenerate and hence

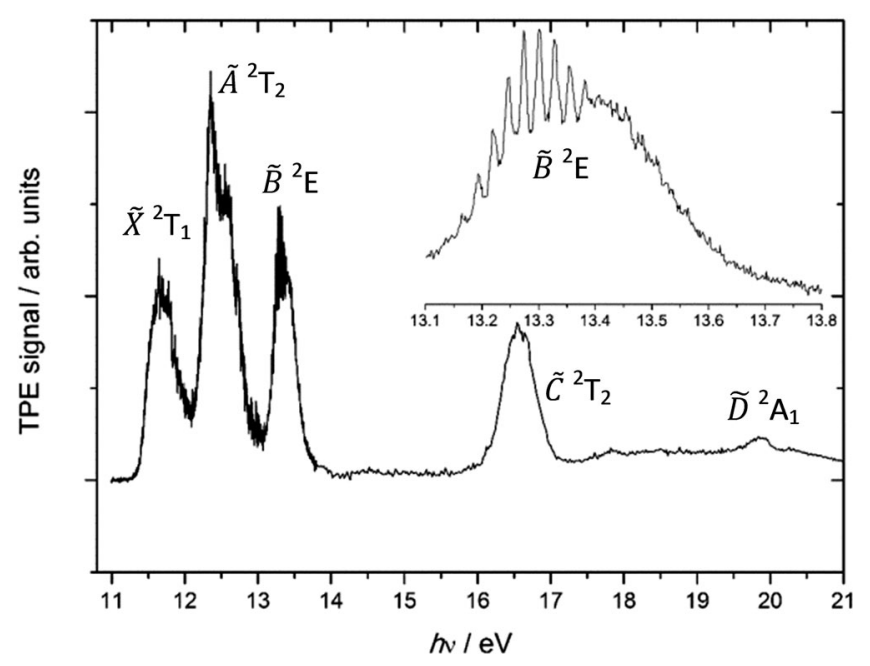

Fig. 1 Threshold photoelectron spectrum of $\mathrm{CCl}_{4}$. The inset shows an enlarged scale TPES of the third band belonging to the B̃ state from 13.1 to $13.8 \mathrm{eV}$ with $0.002 \mathrm{eV}$ resolution.

more intense $G_{3 / 2}$ component has lower energy than the lower degenerate $E_{5 / 2}$ component, with an intensity ratio of $2: 1$, respectively, confirming that the sign of the spin-orbit splitting parameter is negative. ${ }^{38}$ No vibrational structure is observed in the Ã peak. This either indicates a short lifetime and/or large geometry change upon ionisation. To the best of our knowledge, no one has yet reported the vibrational progression clearly seen in the third photoelectron band belonging to the $\tilde{\mathrm{B}}^{2} \mathrm{E}$ state (inset in Fig. 1). The $0.025 \mathrm{eV}\left(200 \mathrm{~cm}^{-1}\right)$ splitting suggests that the $\nu_{2}(e)$ scissors mode is active, as the corresponding harmonic frequency in neutral $\mathrm{CCl}_{4}$ is $214 \mathrm{~cm}^{-1}$ (B3LYP/cc-pVTZ+d result). This means that the geometry change when ionising to the $\tilde{\mathrm{B}}$ state is only moderate, and the Franck-Condon factors for ionisation into the potential energy well are large. Excitation of non-totally-symmetric vibrations such as $\nu_{2}$ of $e$ symmetry are formally forbidden in photoelectron spectroscopy unless the point group of the molecule changes. Therefore, this mode can only gain intensity through Jahn-Teller distortion of the doubly-degenerate ${ }^{2} \mathrm{E}$ electronic state. Herzberg ${ }^{39}$ has shown that the symmetry of the vibration which causes the distortion must be contained in the direct product of the symmetry of the degenerate electronic state with itself. In $T_{\mathrm{d}}$ geometry, $\mathrm{E} \otimes \mathrm{E}=\mathrm{A}_{1}+\mathrm{A}_{2}+\mathrm{E}$, therefore the $\nu_{2}(e)$ mode possesses the correct symmetry to cause JahnTeller distortion. At the same time, internal conversion of $\tilde{B}$ to the shorter-lived $\tilde{A}$ and X̃ states, surely driven by its Jahn-Teller distorted structure, must be slow enough that the $\tilde{B}$-state potential energy surface can support bound nuclear vibrational wave functions.

In the Koopmans picture, these first three states correspond to ionisation from the chlorine $3 \mathrm{p} \pi$ lone pair orbitals with symmetry $e+t_{1}+t_{2}$. The next band in the TPES of $\mathrm{CCl}_{4}$, the $\tilde{\mathrm{C}}{ }^{2} \mathrm{~T}_{2}$ peak, corresponds to ionisation from a $\mathrm{C}-\mathrm{Cl}$ bonding orbital and is centred at $16.58 \mathrm{eV}$, followed by the band corresponding to ionisation to the highest-lying valence electronic state of the parent cation, $\tilde{\mathrm{D}}^{2} \mathrm{~A}_{1}$, centred at $h \nu=19.86 \mathrm{eV}$. 


\section{Breakdown diagram and calculated energetics}

Based on the photoion mass selected threshold photoelectron signal, fractional fragment ion abundances have been determined and are plotted as a function of photon energy in the $14-21 \mathrm{eV}$ range in Fig. 2. As with $\mathrm{CF}_{4}$, it has long been accepted that $\mathrm{CCl}_{4}$ dissociatively photoionises and the intact parent ion can scarcely be detected. ${ }^{21}$ Thus, in agreement with previous reports, ${ }^{31}$ only $\mathrm{CCl}_{3}{ }^{+}$with $100 \%$ fractional abundance was observed in the $\tilde{\mathrm{X}}, \tilde{\mathrm{A}}$, and $\tilde{\mathrm{B}}$ peaks of the spectrum below $14 \mathrm{eV}$. Between 15 and $16 \mathrm{eV}$, the $\mathrm{CCl}_{3}{ }^{+}$signal disappears and signal due to $\mathrm{CCl}_{2}{ }^{+}$rises, only for the heavier $\mathrm{CCl}_{3}{ }^{+}$fragment ion to make a brief and weak return between 17 and $18 \mathrm{eV}$. This observation is strong evidence that dissociation cannot be impulsive in the Franck-Condon gap just below the $\tilde{\mathrm{C}}^{2} \mathrm{~T}_{2}$ peak. If most of the excess energy could be released as kinetic energy in the dissociation, there would always be low internal energy $\mathrm{CCl}_{3}{ }^{+}$intermediates with insufficient energy to dissociate further; this contradicts the negligible $\mathrm{CCl}_{3}{ }^{+}$ signal between 16 and $17 \mathrm{eV}$. For the same reason, the parent ion cannot lose internal energy by fluorescent decay. At higher energies, the $\mathrm{CCl}_{2}^{+}$signal decays and the $50 \%$ crossover energy with $\mathrm{CCl}^{+}$lies at $19.2 \mathrm{eV}$. The breakdown diagram remains unaffected by the presence of the $\tilde{\mathrm{D}}{ }^{2} \mathrm{~A}_{1}$ peak in the TPES at $19.9 \mathrm{eV}$, and the $\mathrm{CCl}^{+}$fractional abundance reaches almost $100 \%$ at $20.5 \mathrm{eV} . \mathrm{C}^{+}, \mathrm{Cl}^{+}$and $\mathrm{Cl}_{2}^{+}$have not been observed in the energy range studied, which is also well below the $27.99 \mathrm{eV}$ double photoionisation threshold (CBS-QB3 calculation to form triplet ground-state $\mathrm{CCl}_{4}{ }^{2+}$ ).

The breakdown diagram can be interpreted in the context of energetically allowed dissociation reactions. Based on the $0 \mathrm{~K}$ heats of formation of $\mathrm{CCl}_{4}, \mathrm{CCl}_{3}{ }^{+}$and $\mathrm{Cl}$ determined from a global fit to a halomethane thermochemical network, ${ }^{22}$ we derive the $\mathrm{CCl}_{4} \rightarrow \mathrm{CCl}_{3}{ }^{+}+\mathrm{Cl}+\mathrm{e}^{-}$threshold at $0 \mathrm{~K}$ to be $11.021 \pm 0.047 \mathrm{eV}$. Furthermore, Rademann et al. ${ }^{41}$ measured $\Delta_{\mathrm{f}} H_{298 \mathrm{~K}}^{\mathrm{o}}\left(\mathrm{CCl}_{2}^{+}\right)=$ $1107.9 \pm 7.5 \mathrm{~kJ} \mathrm{~mol}^{-1}$, which can be converted to $1106.9 \pm$ $7.5 \mathrm{~kJ} \mathrm{~mol}^{-1}$ at $0 \mathrm{~K}$ using the W1U calculated thermal enthalpy of $\mathrm{CCl}_{2}^{+}, 11.18 \mathrm{~kJ} \mathrm{~mol}^{-1}$, and the published thermal enthalpies

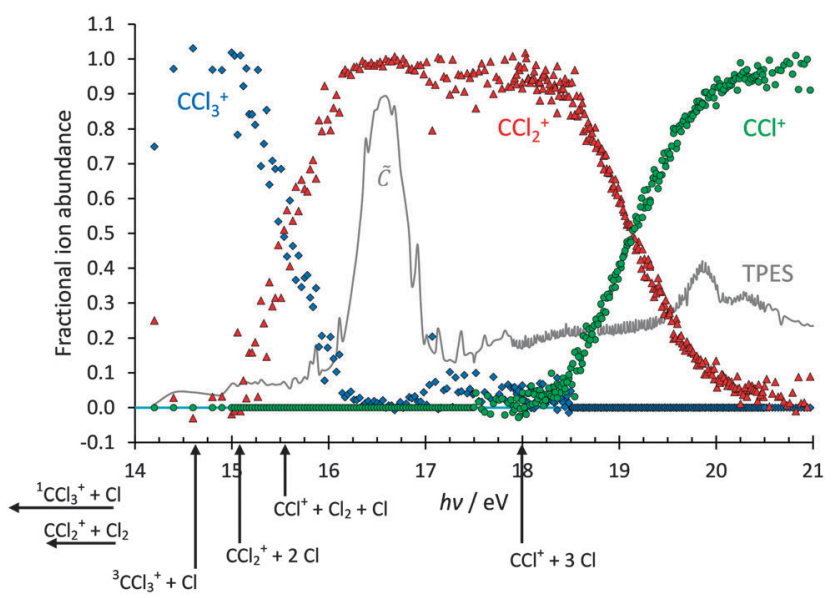

Fig. 2 Breakdown diagram of $\mathrm{CCl}_{4}$ in the 14-21 eV photon energy range. Below $14 \mathrm{eV}$, only $\mathrm{CCl}_{3}{ }^{+}$was observed in the mass spectrum. The W1U calculated $0 \mathrm{~K}$ dissociative photoionisation energies are also shown.
Table 1 Calculated $0 \mathrm{~K}$ dissociative photoionisation energies of $\mathrm{CCl}_{4}$

\begin{tabular}{|c|c|c|c|c|}
\hline \multirow[b]{2}{*}{$\mathrm{CCl}_{4}-\mathrm{e}^{-} \rightarrow$} & \multicolumn{4}{|l|}{$E_{0} / \mathrm{eV}$} \\
\hline & CBS-QB3 & G4 & W1U & Literature $^{a}$ \\
\hline${ }^{1} \mathrm{CCl}_{3}^{+}+\mathrm{Cl}^{b}$ & 11.154 & 11.057 & 11.074 & $11.021 \pm 0.047$ \\
\hline${ }^{3} \mathrm{CCl}_{3}^{+}+\mathrm{Cl}$ & 14.658 & 14.693 & 14.711 & \\
\hline $\mathrm{CCl}_{2}^{+}+2 \mathrm{Cl}$ & 15.199 & 15.055 & 15.087 & $14.926 \pm 0.085$ \\
\hline $\mathrm{CCl}_{2}^{+}+\mathrm{Cl}_{2}$ & 12.719 & 12.576 & 12.607 & \\
\hline $\mathrm{CCl}^{+}+3 \mathrm{Cl}$ & 18.126 & 18.006 & 18.015 & \\
\hline $\mathrm{CCl}^{+}+\mathrm{Cl}_{2}+\mathrm{Cl}$ & 15.647 & 15.526 & 15.535 & \\
\hline
\end{tabular}

${ }^{a}$ See text for derivation. ${ }^{b}$ The $\mathrm{Cl}_{2}$ energy was calculated, and the $\mathrm{Cl}$ energy was obtained as half the $\mathrm{Cl}_{2}$ energy plus the $0 \mathrm{~K} 1 / 2 \mathrm{Cl}_{2} \rightarrow \mathrm{Cl}$ reaction energy of $119.6 \mathrm{~kJ} \mathrm{~mol}^{-1} . \mathrm{.0}^{\circ}$

of $\mathrm{C}$ and $\mathrm{Cl}_{2}$ of 1.05 and $9.18 \mathrm{~kJ} \mathrm{~mol}^{-1},{ }^{40}$ respectively. Thus, the $0 \mathrm{~K}$ dissociative photoionisation threshold for $\mathrm{CCl}_{4} \rightarrow \mathrm{CCl}_{2}^{+}+$ $2 \mathrm{Cl}+\mathrm{e}^{-}$lies at $14.926 \pm 0.085 \mathrm{eV}$. In order to confirm these values, we have calculated the thresholds for dissociative photoionisation of $\mathrm{CCl}_{4}$ to form $\mathrm{CCl}_{3}{ }^{+}, \mathrm{CCl}_{2}{ }^{+}$and $\mathrm{CCl}^{+}$, including the formation of the lowest triplet (excited) state of $\mathrm{CCl}_{3}{ }^{+}$, using the CBS-QB3, G4 and W1U composite methods. The results are summarized in Table 1.

The agreement between computational methods and literature data is reassuringly good. Furthermore, the rise of the $\mathrm{CCl}_{2}^{+}$and $\mathrm{CCl}^{+}$signals (at ca. 15.0 and $18.1 \mathrm{eV}$, respectively) in the breakdown diagram corresponds to the calculated thermochemical onsets of $2 \mathrm{Cl}$ and $3 \mathrm{Cl}$ losses. Because of the uncertainty in some of the energetics and the fact that the $\mathrm{CCl}_{2}{ }^{+}$ appearance energy is in a Franck-Condon gap, inaccessible with non-threshold ionisation, previously the possibility of $\mathrm{Cl}_{2}$ formation could not be ruled out completely. ${ }^{21,42}$ Based on these results, we can now say that no $\mathrm{Cl}_{2}$ loss takes place below the $\mathrm{CCl}_{2}^{+}+2 \mathrm{Cl}+\mathrm{e}^{-}$or $\mathrm{CCl}^{+}+3 \mathrm{Cl}+\mathrm{e}^{-}$thresholds. Nonetheless, preliminary reaction path calculations, similar to the Cl-loss channels discussed in the next section, indicate that there may be unbound electronic states of $\mathrm{CCl}_{4}{ }^{+}$leading to production of $\mathrm{CCl}_{2}{ }^{+}+\mathrm{Cl}_{2}$. While the smaller-than-expected kinetic energy release (see breakdown diagram fits below) indicates that these electronic states do not play a significant role in the dissociative photoionisation mechanism, they may play a minor role above the $\mathrm{CCl}_{2}{ }^{+}+2 \mathrm{Cl}+\mathrm{e}^{-}$and $\mathrm{CCl}^{+}+3 \mathrm{Cl}+\mathrm{e}^{-}$thresholds. However, based on the computed energetics and the appearance of the breakdown diagram, we can assume that little if any $\mathrm{Cl}_{2}$ is produced in the valence dissociative photoionisation of $\mathrm{CCl}_{4}$.

\section{Cl-loss potential energy curves, $\mathrm{CCl}_{4}{ }^{+} \rightarrow \mathrm{CCl}_{3}{ }^{+}+\mathrm{Cl}$}

Notwithstanding symmetry, the electronic ground state potential energy surface of $\mathrm{CCl}_{4}{ }^{+}$is a function of nine internal coordinates. To address fully the Cl-loss reaction on the ground and electronic excited states of $\mathrm{CCl}_{4}{ }^{+}$, the electronic degree of freedom and spin-orbit effects would have to be included. Evidently, the computational problem needs to be simplified to become tractable. First, we have restricted the symmetry of the geometries along the reaction coordinate to $C_{3 \mathrm{v}}$ to correspond to that of the products. There are then only three internal coordinates: the $\mathrm{C}-\mathrm{Cl}$ bond length in the $\mathrm{CCl}_{3}{ }^{+}$fragment ion, the $\mathrm{C}-\mathrm{Cl}$ bond length in the breaking bond, and the angle between the 


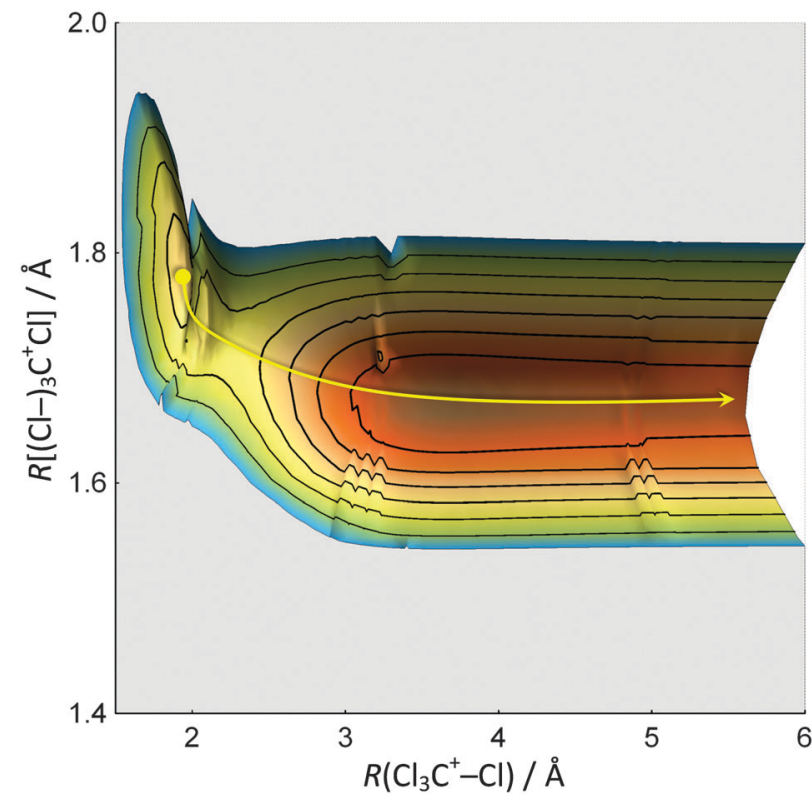

Fig. 3 Ground electronic state potential energy surface of $\mathrm{Cl}$-loss from $\mathrm{CCl}_{4}{ }^{+}$in $\mathrm{C}_{3 \mathrm{v}}$ symmetry, shown from the energy minimum up to $1 \mathrm{eV}$. The $T_{\mathrm{d}}$ structure at a bond length of about $1.8 \AA$ corresponds to a local minimum in $C_{3 v}$ symmetry. The loss of a $\mathrm{Cl}$ is simultaneous with the contraction of the other $\mathrm{C}-\mathrm{Cl}$ bonds, and proceeds over a small barrier to yield the minimum energy $\mathrm{CCl}_{3}{ }^{+}+\mathrm{Cl}$ products. The minimum energy path on this surface is also shown.

$\mathrm{C}-\mathrm{Cl}$ bonds in $\mathrm{CCl}_{3}{ }^{+}$with the rotational symmetry axis which converges to $90^{\circ}$ as the $\mathrm{Cl}$ leaves and planar ground-state singlet ${ }^{1} \mathrm{CCl}_{3}{ }^{+}$is formed. The two bond lengths were scanned and the bond angle optimized to obtain the ground state potential energy surface of $\mathrm{CCl}_{4}^{+}$as shown in Fig. 3. The Cl-loss reaction coordinate was then defined as the minimum energy path connecting the shallow tetrahedral minimum in $C_{3 v}$ symmetry with the ${ }^{1} \mathrm{CCl}_{3}{ }^{+}+\mathrm{Cl}$ products. Thereafter, the ground and excited doublet state energies were calculated along this path using EOM-IP-CCSD, and the lowest quartet state curve was obtained by calculating the doublet-quartet splitting using MP2 and adding it to the doublet ground-state energy. The results are shown in Fig. 4.

The triply-degenerate $\tilde{\mathrm{X}}^{2} \mathrm{~T}_{1}$ state of $\mathrm{CCl}_{4}{ }^{+}$splits into a doublydegenerate unbound and a singly-degenerate bound state along the reaction coordinate. On the other hand, the triply degenerate $\tilde{\mathrm{A}}^{2} \mathrm{~T}_{2}$ state splits into a doubly-degenerate bound and a singlydegenerate repulsive state; the latter state is degenerate with the repulsive component of the $\tilde{X}$ state. Strictly speaking, these states are not purely repulsive. The lowest lying ion curve appears to be bound at tetrahedral geometry but the potential well is probably insufficient to support a vibrational state. Further out, the electronic energy plateaus and leads to a second shallow minimum at $c a .3 .25 \AA$ A However, in the Franck-Condon envelope, the internal energy of the ion is more than sufficient to lead to prompt dissociation. At higher energies, the $\tilde{\mathrm{B}}{ }^{2} \mathrm{E}$ and $\tilde{\mathrm{C}}^{2} \mathrm{~T}_{2}$ doublet states of $\mathrm{CCl}_{4}{ }^{+}$appear to be bound and do not correlate with low-energy $\mathrm{CCl}_{3}{ }^{+}+\mathrm{Cl}$ products. These dissociative potential energy surfaces are consistent with the absence of vibrational

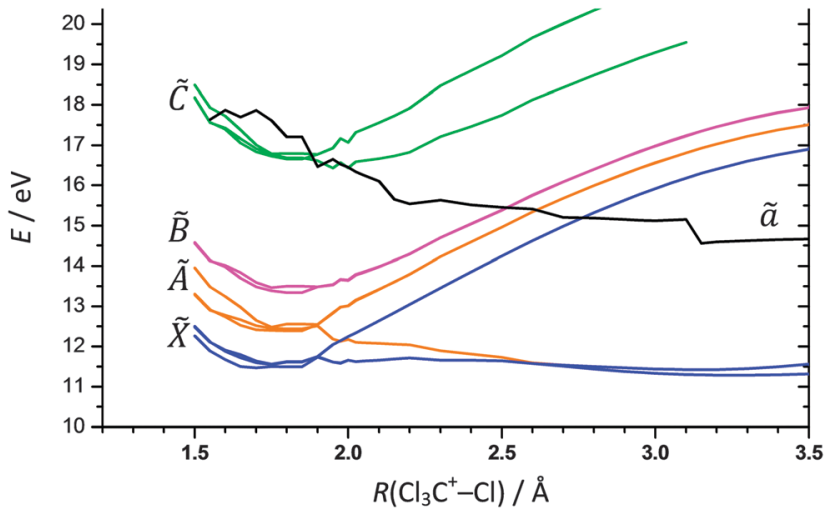

Fig. 4 Calculated potential energy curves for the ground and first three excited doublet and the lowest quartet states along the $\mathrm{Cl}_{3} \mathrm{C}^{+}-\mathrm{Cl}$ dissociation coordinate.

fine structure in the first two bands of the TPES of $\mathrm{CCl}_{4}$ and the observation of the $\nu_{2}(e)$ scissors mode in the $\tilde{\mathrm{B}}{ }^{2} \mathrm{E}$ state. The calculated potential energy curve is not as smooth for the lowest quartet state of $\mathrm{CCl}_{4}{ }^{+}$, but it shows an intersection with the $\tilde{\mathrm{C}}^{2} \mathrm{~T}_{2}$ state around $16 \mathrm{eV}$. Furthermore, the quartet state leads to the production of triplet ${ }^{3} \mathrm{CCl}_{3}{ }^{+}+\mathrm{Cl}$. Keeping in mind that the $\tilde{\mathrm{B}}{ }^{2} \mathrm{E}$ state, but not the $\tilde{\mathrm{C}}^{2} \mathrm{~T}_{2}$ state, of $\mathrm{CCl}_{4}{ }^{+}$supports bound vibrational wave functions, there is some evidence from the TPES that, among the first four doublet $\mathrm{CCl}_{4}{ }_{4}$ states, $\tilde{\mathrm{B}}^{2} \mathrm{E}$ is the longest lived. Smith et al. proposed that $\mathrm{CCl}_{4}{ }^{+}$behaves analogously to $\mathrm{CF}_{4}{ }^{+}$, suggesting that dissociation from the $\tilde{\mathrm{B}}^{2} \mathrm{E}$ state occurs via internal conversion into the $\tilde{\mathrm{A}}{ }^{2} \mathrm{~T}_{2}$ state. ${ }^{42}$ This conclusion is supported by the experimental and computational results reported here.

\section{Statistical modelling and dissociation mechanism}

In a statistical dissociation, the excess energy, i.e. the energy available to the system above the dissociation threshold, is partitioned among the internal degrees of freedom of the fragments and their translational kinetic energies according to the respective phase space volumes. ${ }^{7,43}$ Aside from spin-orbit and electronically excited states, the chlorine atom cannot accommodate internal energy, therefore most of the excess energy is expected to stay in the fragment ion produced from $\mathrm{CCl}_{4}{ }^{+}$. In terms of which vibrational modes of $\mathrm{CCl}_{3}{ }^{+}$might be excited, one would only expect the umbrella motion and the symmetric stretch to be active, as the reaction coordinate is a combination of $\mathrm{C}-\mathrm{Cl}$ bond contraction, $\mathrm{Cl}_{3} \mathrm{C}^{+}-\mathrm{Cl}$ bond elongation, and increasing planarity of $\mathrm{CCl}_{3}{ }^{+}$(see also previous section). ${ }^{44}$ During impulsive dissociations a significant amount of the excess energy can be released as kinetic energy of the fragments, ${ }^{45}$ as the force and the resulting acceleration along the reaction coordinate is such that the internal energy cannot be redistributed quickly enough before fragmentation is complete. High kinetic energy release leads to low internal energy fragments, which may not have enough energy to dissociate further, and are stable well above their dissociative photoionisation energies.

As mentioned earlier, the breakdown diagram of $\mathrm{CCl}_{4}$ in the 14-21 eV photon energy range (Fig. 2), observed for the first time with better than $10 \mathrm{meV}$ internal energy resolution and 
good signal-to-noise ratio, appears to tell a different story from that of an impulsive dissociation. The $\mathrm{CCl}_{3}{ }^{+} / \mathrm{CCl}_{2}{ }^{+}$and $\mathrm{CCl}_{2}{ }^{+}$/ $\mathrm{CCl}^{+}$crossovers are quite sharp, and do not suggest suprastatistical kinetic energy release. The weak but reproducible recurrence of the $\mathrm{CCl}_{3}{ }^{+}$signal between 17 and $18 \mathrm{eV}$ indicates a decrease in the $\mathrm{CCl}_{3}{ }^{+}$internal energy as the photon energy increases, and suggests that there are two dissociation mechanisms at play.

Unlike the equivalent $\tilde{\mathrm{C}}^{2} \mathrm{~T}_{2}$ state of $\mathrm{CF}_{4}{ }^{+}, \mathrm{SiCl}_{4}{ }^{+}$and $\mathrm{GeCl}_{4}{ }^{+}$ where fluorescence is a major decay channel with lifetimes of 9 , 38 and $65 \mathrm{~ns}$, respectively, ${ }^{46,47}$ the $\tilde{\mathrm{C}}^{2} \mathrm{~T}_{2}$ state of $\mathrm{CCl}_{4}{ }^{+}$does not appear to decay radiatively. Its apparently short, sub-ns lifetime, together with an available intersystem crossing path onto the lowest (ã) quartet state which dissociates to triplet ${ }^{3} \mathrm{CCl}_{3}{ }^{+}$, offers an alternative dissociation path to fluorescence, direct dissociation, or internal conversion to lower doublet states. Because of the different level spacing and the required closeness of the $\tilde{C}$ and ã states in energy near the minimum of the $\tilde{\mathrm{B}}$ state, such a fast dissociative decay route as opposed to radiative decay may be unique to $\mathrm{CCl}_{4}{ }^{+}$within this family of compounds. Furthermore, such fast intersystem crossing paths have been detected in water, ${ }^{9,48}$ and the larger spin-orbit coupling in $\mathrm{CCl}_{4}{ }^{+}$makes them just as plausible here. If the $\tilde{\mathrm{C}}$ ${ }^{2} \mathrm{~T}_{2}$ state of $\mathrm{CCl}_{4}{ }^{+}$decays in this way, the energy of the singlettriplet gap in $\mathrm{CCl}_{3}{ }^{+}$of $\mathrm{ca}$. $3.6 \mathrm{eV}$ (Table 1), will be 'trapped' in the fragment ion and be unavailable for kinetic energy release. If singlet $\mathrm{CCl}_{3}{ }^{+}$production is allowed again at higher energies, the effective internal energy of the fragment ion may indeed decrease with increasing photon energy, as appears to be observed in our experiment.

We have modelled the breakdown diagram (Fig. 2) assuming the W1U-determined onset energies (Table 1), two dimensional kinetic energy release, ${ }^{49}$ and, consistent with symmetric daughter ion peak shapes, fast sequential dissociations. ${ }^{7}$ The calculated breakdown diagram is shown in Fig. 5(a) for singlet, and in Fig. 5(b) triplet $\mathrm{CCl}_{3}{ }^{+}$being the intermediate ion. The $\mathrm{CCl}_{2}{ }^{+}$ signal is reproduced well in Fig. 5(a) at its onset in the FranckCondon gap at $15 \mathrm{eV}$, albeit at a slightly lower appearance energy than observed. The calculated curve disagrees with the measured one more as we approach the $\tilde{\mathrm{C}}^{2} \mathrm{~T}_{2}$ peak in the photoelectron spectrum. We propose that the reason for this disagreement is $\tilde{\mathrm{C}}^{2} \mathrm{~T}_{2}$ state Rydberg series involvement below ca. $16 \mathrm{eV}$. As observed for the dissociation mechanism change in F-atom loss in the 1,1-difluoroethene cation, ${ }^{14}$ Rydberg states may behave similarly to their convergent ionic state also regarding their dissociative decay. In $\mathrm{CCl}_{4}^{+}$, this means that $\tilde{\mathrm{C}}$ state Rydberg series may autoionise to the quartet ã state at geometries where the two are close to degenerate. Cl loss will then yield triplet ${ }^{3} \mathrm{CCl}_{3}{ }^{+}$. As the photon energy reaches $16 \mathrm{eV}$, which corresponds to the onset of the $\tilde{\mathrm{C}}^{2} \mathrm{~T}_{2}$ ion state, the fractional abundance of $\mathrm{CCl}_{2}^{+}$rises sharply at the cost of that of $\mathrm{CCl}_{3}{ }^{+}$. This indicates that the photoionisation mechanism is completely dominated by the $\tilde{\mathrm{C}}$ state, which leads to the production of triplet-state ${ }^{3} \mathrm{CCl}_{3}{ }^{+}$fragments. The singlet-triplet excitation energy stays trapped in the fragment ion, which limits the excess energy release in the first Cl-loss channel. (a)

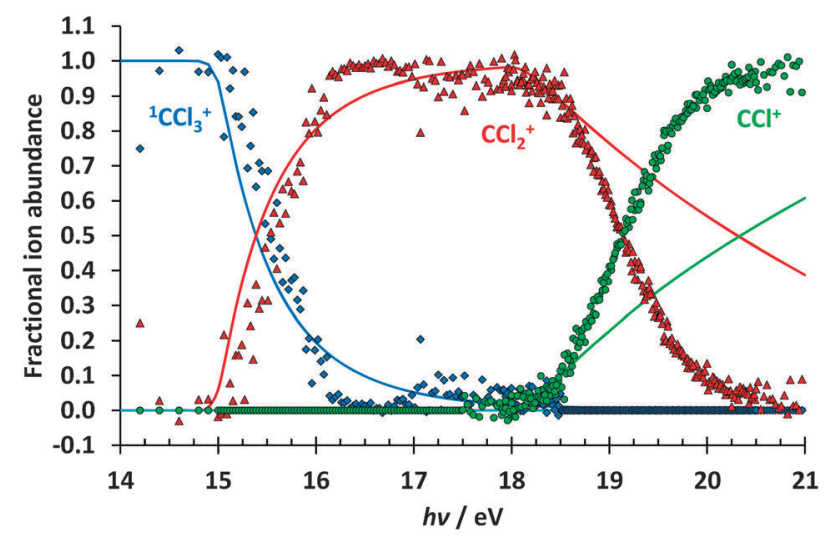

(b)

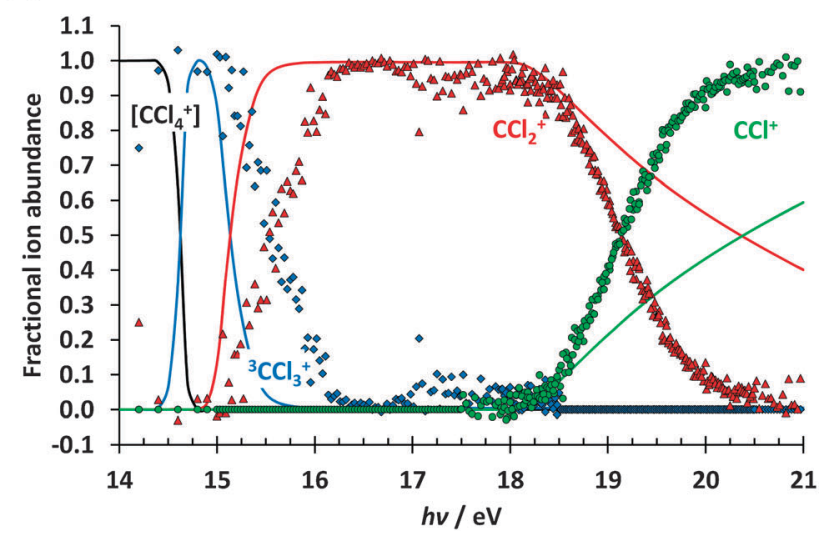

Fig. 5 Calculated $\mathrm{CCl}_{4}$ breakdown diagram assuming statistical and fast, $k\left(E_{0}\right)>10^{7} \mathrm{~s}^{-1}$, dissociation, using ab initio onsets for (a) singlet and (b) triplet $\mathrm{CCl}_{3}{ }^{+}$formation. The dots are the experimentally measured fractional ion abundances, continuous lines correspond to the model calculation.

With less kinetic energy being available for release and more excess energy trapped in the system, it is more likely to be sufficiently energetic to lose a further $\mathrm{Cl}$ to form $\mathrm{CCl}_{2}{ }^{+}$. The limited regeneration of the $\mathrm{CCl}_{3}{ }^{+}$signal at $c a .17 \mathrm{eV}$, past the Franck-Condon zone of the $\tilde{\mathrm{C}}^{2} \mathrm{~T}_{2}$ state, is again well reproduced by the assumption that the intermediate is singlet ${ }^{1} \mathrm{CCl}_{3}{ }^{+}$, again suggesting autoionisation of these states to the $\tilde{\mathrm{B}}^{2} \mathrm{E}$ state prior to singlet ${ }^{1} \mathrm{CCl}_{3}{ }^{+}$formation on the $\tilde{\mathrm{A}}$ or $\tilde{\mathrm{X}}$ state potential energy surface. In summary, the triplet state involvement is inferred chiefly from the disappearance of the $\mathrm{CCl}_{3}{ }^{+}$signal in the energy range of the $\tilde{\mathrm{C}}$ state and its faint and fleeting reapparition at slightly higher energies.

The onset of the $\mathrm{CCl}^{+}$signal is also relatively well reproduced by the statistical model, but since the overall excess energy is independent of the intermediate energy, the shape of the breakdown curve is hardly affected by the choice of $\mathrm{CCl}_{3}{ }^{+}$spin state. Above $18.5 \mathrm{eV}$, the excess energy released is actually less than predicted by statistical theory, and the $\mathrm{CCl}^{+}$abundance rises more quickly with increasing photon energy. Such discrepancies are not unheard of at high excess energies, ${ }^{50}$ but its magnitude here suggests possible electronically excited $\mathrm{CCl}_{2}{ }^{+}$ participation, similarly to the triplet $\mathrm{CCl}_{3}{ }^{+}$intermediates. 
At the same time, the $\mathrm{W} 1 \mathrm{U}$ onset energy of $\mathrm{CCl}^{+}$with three $\mathrm{Cl}$ atoms is confirmed by the well-reproduced rising edge of the $\mathrm{CCl}^{+}$breakdown curve.

\section{Conclusions}

We have revisited the dissociative photoionisation of $\mathrm{CCl}_{4}$ between its ionisation energy and $21 \mathrm{eV}$, covering the five valence ion states. Composite calculations were carried out to confirm the dissociative photoionisation onset energies. The minimum energy Cl-loss path in $\mathrm{CCl}_{4}{ }^{+}$to singlet ${ }^{1} \mathrm{CCl}_{3}{ }^{+}+\mathrm{Cl}$ was found by a 2-dimensional potential energy scan, and electronic excited state energies in the parent cation were calculated using EOM-IP-CCSD. We have found a direct path from the $\tilde{\mathrm{A}}^{2} \mathrm{~T}_{2}$ state of $\mathrm{CCl}_{4}{ }^{+}$to the promptly-dissociating $\tilde{\mathrm{X}}^{2} \mathrm{~T}_{1}$ state, which explains the lack of vibrational structure in the TPES of both bands. The $\tilde{\mathrm{B}}{ }^{2} \mathrm{E}$ state, on the other hand, supports bound vibrational wave functions, shown by a progression being observed in the $\nu_{2}(e)$ scissors mode in this band of the TPES. This mode can only show activity in a photoelectron spectrum of a tetrahedral molecule if the symmetry is broken. The ${ }^{2} \mathrm{E}$ electronic state of the ion is doubly degenerate. Since $\mathrm{E} \subset \mathrm{E} \otimes \mathrm{E}$ in $T_{\mathrm{d}}$, the vibration has the correct $e$ symmetry to induce Jahn-Teller distortion from $T_{\mathrm{d}}$ symmetry; the vibration then becomes totally symmetric in the lower-symmetry $D_{2 \mathrm{~d}}$ point group, making the vibrational progression observable.

A statistical model reproduces the $\mathrm{CCl}_{2}^{+}$fractional abundance in the breakdown curve at the $\mathrm{CCl}_{2}{ }^{+}+2 \mathrm{Cl}+\mathrm{e}^{-}$threshold well, which is further evidence for the presence of a long-lived $\mathrm{CCl}_{4}{ }^{+}$intermediate undergoing IVR at higher photon energies. However, the $\mathrm{CCl}_{3}{ }^{+}$signal then drops rapidly in the vicinity of the $\tilde{\mathrm{C}}^{2} \mathrm{~T}_{2}$ band of $\mathrm{CCl}_{4}{ }^{+}$. An intersystem crossing pathway via the lowest quartet state yielding triplet ${ }^{3} \mathrm{CCl}_{3}{ }^{+}+\mathrm{Cl}$ is proposed to be the reason. By decreasing the excess energy available for kinetic energy release, this allows for quantitative $\mathrm{CCl}_{2}{ }^{+}$production over the energy range of the $\tilde{\mathrm{C}}^{2} \mathrm{~T}_{2}$ band. $\mathrm{CCl}_{3}{ }^{+}$makes a weak return in the breakdown diagram to high energy of the $\tilde{\mathrm{C}}$ peak, which is reproduced by the statistical 'singlet- $\mathrm{CCl}_{3}{ }^{+}$, model. At a higher energy of $c a .18 \mathrm{eV}, \mathrm{CCl}^{+}$is produced from the $\mathrm{CCl}_{2}{ }^{+}$cation. The onset of this process is predicted by the statistical model well, but the $\mathrm{CCl}^{+}$signal rises more steeply than predicted shortly after its onset, indicating suppressed kinetic energy release.

In conclusion, there are non-statistical aspects to the dissociative photoionisation of $\mathrm{CCl}_{4}^{+}$in the $\tilde{\mathrm{C}}^{2} \mathrm{~T}_{2}$ band, which increase the internal energy available for sequential $\mathrm{Cl}$ losses, but the breakdown curves in the onset region for production of the $\mathrm{CCl}_{2}{ }^{+}$and $\mathrm{CCl}^{+}$daughter ions are both described well by a statistical model. When the initial ion state is the $\tilde{\mathrm{B}}$ state or higher, the dissociative ground and first excited electronic states of $\mathrm{CCl}_{4}^{+}$are only accessed by internal conversion after IVR has taken place. As triplet-state ${ }^{3} \mathrm{CCl}_{3}{ }^{+}$production recedes to higher energy of the $\tilde{\mathrm{C}}^{2} \mathrm{~T}_{2}$ band in the TPES, the internal energy available for dissociation actually decreases with increasing photon energy, resulting in a brief re-appearance of the $\mathrm{CCl}_{3}{ }^{+}$signal.
This behaviour is in contrast with that of some of the fluoroethene cations, in which the dissociative photoionisation starts out as a statistical process and then exhibits non-statistical F-loss, or, in the case of $\mathrm{C}_{2} \mathrm{~F}_{4}{ }^{+}$, a second, de-coupled statistical dissociation regime. ${ }^{13,14}$ The parent cation of tetrachloromethane dissociates impulsively at low energies, but then behaves almost statistically at higher energies. The decay of the $\tilde{\mathrm{C}}{ }^{2} \mathrm{~T}_{2}$ state of $\mathrm{CCl}_{4}{ }^{+}$ions also appears to be distinctively different from that of analogous $\tilde{\mathrm{C}}$ state of other $\mathrm{MX}_{4}{ }^{+}$ions, which have previously been reported to be quite long-lived and decay by fluorescence. The opportune quasi-degeneracy of the doublet $\tilde{\mathrm{C}}$ and quartet ã states in $\mathrm{CCl}_{4}{ }^{+}$therefore seems unlikely to occur in the other members of this family of compounds.

\section{Acknowledgements}

The iPEPICO experiments were carried out at the VUV beamline of the Swiss Light Source of the Paul Scherrer Institut. The research leading to these results has received funding from the European Community's Seventh Framework Programme (FP7/2007-2013) under grant agreement no. 226716. JH thanks the University of Birmingham for a Research Studentship.

\section{Notes and references}

1 T. I. Sølling, T. S. Kuhlman, A. B. Stephansen, L. B. Klein and K. B. Møller, ChemPhysChem, 2014, 15, 249-259.

2 S. Ullrich, T. Schultz, M. Z. Zgierski and A. Stolow, Phys. Chem. Chem. Phys., 2004, 6, 2796.

3 Y. Liu, G. Knopp, P. Hemberger, Y. Sych, P. Radi, A. Bodi and T. Gerber, Phys. Chem. Chem. Phys., 2013, 15, 18101-18107.

4 T. Baer, B. Sztaray, J. P. Kercher, A. F. Lago, A. Bodi, C. Skull and D. Palathinkal, Phys. Chem. Chem. Phys., 2005, 7, 1507-1513.

5 M. Johnson, A. Bodi, L. Schulz and T. Gerber, Nucl. Instrum. Methods Phys. Res., Sect. A, 2009, 610, 597-603.

6 A. Bodi, M. Johnson, T. Gerber, Z. Gengeliczki, B. Sztaray and T. Baer, Rev. Sci. Instrum., 2009, 80, 34101.

7 B. Sztáray, A. Bodi and T. Baer, J. Mass Spectrom., 2010, 45, 1233-1245.

8 A. Bodi, P. Hemberger and T. Gerber, J. Chem. Thermodyn., 2013, 58, 292-299.

9 A. Bodi, J. Csontos, M. Kállay, S. Borkar and B. Sztáray, Chem. Sci., 2014, 5, 3057-3063.

10 U. Lourderaj and W. L. Hase, J. Phys. Chem. A, 2009, 113, 2236-2253.

11 T. Baer and A. R. Potts, J. Phys. Chem. A, 2000, 104, 9397-9402.

12 A. Bodi, N. S. Shuman and T. Baer, Phys. Chem. Chem. Phys., 2009, 11, 11013-11021.

13 J. Harvey, A. Bodi, R. P. Tuckett and B. Sztaray, Phys. Chem. Chem. Phys., 2012, 14, 3935-3948.

14 J. Harvey, P. Hemberger, A. Bodi and R. P. Tuckett, J. Chem. Phys., 2013, 138, 124301.

15 J. P. Maier, Acc. Chem. Res., 1982, 15, 18-23. 
16 D. M. Smith, R. P. Tuckett, K. R. Yoxall, K. Codling, P. A. Hatherly, J. F. M. Aarts and M. Stankiewicz, J. Chem. Phys., 1994, 101, 10559-10575.

17 P. M. Guyon, T. Baer and I. Nenner, J. Chem. Phys., 1983, 78, 3665-3672.

18 L. A. Shadoff, L. Prókai, G. B. Anderson, R. G. Gills, Q. N. Porter, L. Bencze, G. W. Dillow, I. K. Gregor, P. R. Ashton and M. E. Rose, Org. Mass Spectrom., 1986, 21, 381-390.

19 C. E. C. A. Hop, J. L. Holmes, F. P. Lossing and J. K. Terlouw, Int. J. Mass Spectrom. Ion Processes, 1988, 83, 285-294.

20 J. C. Creasey, H. M. Jones, D. M. Smith, R. P. Tuckett, P. A. Hatherly, K. Codling and I. Powis, Chem. Phys., 1993, 174, 441-452.

21 T. Kinugawa, Y. Hikosaka, A. M. Hodgekins and J. H. D. Eland, J. Mass Spectrom., 2002, 37, 854-857.

22 J. Harvey, R. P. Tuckett and A. Bodi, J. Phys. Chem. A, 2012, 116, 9696-9705.

23 A. T. J. B. Eppink and D. H. Parker, Rev. Sci. Instrum., 1997, 68, 3477.

24 G. A. Garcia, H. Soldi-Lose and L. Nahon, Rev. Sci. Instrum., 2009, 80, 23102.

25 X. F. Tang, X. G. Zhou, M. L. Niu, S. L. Liu, J. D. Sun, X. B. Shan, F. Y. Liu and L. S. Sheng, Rev. Sci. Instrum., 2009, 80, 113101.

26 A. Bodi, B. Sztaray, T. Baer, M. Johnson and T. Gerber, Rev. Sci. Instrum., 2007, 78, 84102.

27 G. A. Garcia, B. K. Cunha de Miranda, M. Tia, S. Daly and L. Nahon, Rev. Sci. Instrum., 2013, 84, 053112.

28 A. Bodi, P. Hemberger, T. Gerber and B. Sztáray, Rev. Sci. Instrum., 2012, 83, 083105.

29 T. Baer, A. Guerrero, J. Z. Davalos and A. Bodi, Phys. Chem. Chem. Phys., 2011, 13, 17791-17801.

30 S. Borkar, B. Sztáray and A. Bodi, Phys. Chem. Chem. Phys., 2011, 13, 13009-13020.

31 J. C. Creasy, I. R. Lambert, R. P. Tuckett, K. Codling, L. J. Frasinski, P. A. Hatherly, M. Stankiewicz and D. M. P. Holland, J. Chem. Phys., 1990, 93, 3295-3306.

32 B. Sztaray and T. Baer, Rev. Sci. Instrum., 2003, 74, 3763-3768.

33 A. Bodi and P. Hemberger, Phys. Chem. Chem. Phys., 2014, 16, 505-515.

34 M. J. Frisch, G. W. Trucks, H. B. Schlegel, G. E. Scuseria, M. A. Robb, J. R. Cheeseman, G. Scalmani, V. Barone, B. Mennucci, G. A. Petersson, S. Nakatsuji, M. Caricato, H. P. Hratchian, A. F. Izmaylov, J. Bloino, G. Zheng, J. L. Sonnenberg, M. Hada, M. Ehara, K. Toyota, R. Fukuda, J. Hasegawa, M. Ishida, T. Nakajima, Y. Honda, O. Kitao, H. Nakai, T. Vreven, J. A. J. A. Montgomery, J. E. Peralta, F. Ogliaro, M. Bearpark, J. J. Heyd, E. Brothers, K. N. Kudin, V. N. Staroverov, R. Kobayashi, J. Normand, K. Raghavachari, A. Rendell, J. C. Burant, S. S. Iyengar, J. Tomasi, M. Cossi, N. Rega, N. J. Millam, M. Klene, J. E. Knox, J. B. Cross, V. Bakken, C. Adamo, J. Jaramillo, R. Gomperts, R. E. Stratmann, O. Yazyev,
A. J. Austin, R. Cammi, C. Pomelli, J. W. Ochterski, R. L. Martin, K. Morokuma, V. G. Zakrzewski, G. A. Voth, P. Salvador, J. J. Dannenberg, S. Dapprich, A. D. Daniels, O. Farkas, J. B. Foresman, J. V Ortiz, J. Cioslowski, D. J. Fox, H. Nakatsuji, X. Li and J. N. Millam, Gaussian 09, Revision C.1, Gaussian, Inc., Wallingford, CT, 2009.

35 Y. Shao, L. F. Molnar, Y. Jung, J. Kussmann, C. Ochsenfeld, S. T. Brown, A. T. B. Gilbert, L. V. Slipchenko, S. V. Levchenko, D. P. O’Neill, R. A. DiStasio, R. C. Lochan, T. Wang, G. J. O. Beran, N. A. Besley, J. M. Herbert, C. Y. Lin, T. Van Voorhis, S. H. Chien, A. Sodt, R. P. Steele, V. A. Rassolov, P. E. Maslen, P. P. Korambath, R. D. Adamson, B. Austin, J. Baker, E. F. C. Byrd, H. Dachsel, R. J. Doerksen, A. Dreuw, B. D. Dunietz, A. D. Dutoi, T. R. Furlani, S. R. Gwaltney, A. Heyden, S. Hirata, C.-P. Hsu, G. Kedziora, R. Z. Khalliulin, P. Klunzinger, A. M. Lee, M. S. Lee, W. Liang, I. Lotan, N. Nair, B. Peters, E. I. Proynov, P. A. Pieniazek, Y. M. Rhee, J. Ritchie, E. Rosta, C. D. Sherrill, A. C. Simmonett, J. E. Subotnik, H. L. Woodcock, W. Zhang, A. T. Bell, A. K. Chakraborty, D. M. Chipman, F. J. Keil, A. Warshel, W. J. Hehre, H. F. Schaefer, J. Kong, A. I. Krylov, P. M. W. Gill and M. Head-Gordon, Phys. Chem. Chem. Phys., 2006, 8, 3172-3191.

36 J. F. Stanton and J. Gauss, J. Chem. Phys., 1994, 101, 8938. 37 W. Von Niessen and L. Asbrink, J. Electron Spectrosc. Relat. Phenom., 1982, 26, 173.

38 R. N. Dixon and R. P. Tuckett, Chem. Phys. Lett., 1987, 140, 553-557.

39 G. Herzberg, Molecular Spectra and Molecular Structure III. Electronic Spectra and Electronic Structure of Polyatomic Molecules, Van Nostrand, Princeton, 1966.

40 M. W. Chase, NIST-JANAF Thermochemical Tables, American Institute of Physics, New York, 1998.

41 K. Rademann, H. W. Jochims and H. Baumgaertel, J. Phys. Chem., 1985, 89, 3459-3464.

42 D. M. Smith, R. P. Tuckett, K. R. Yoxall, K. Codling and P. A. Hatherly, Chem. Phys. Lett., 1993, 216, 493-502.

43 B. Sztáray and T. Baer, J. Phys. Chem. A, 2002, 106, 8046-8053.

44 I. Powis, Mol. Phys., 1980, 39, 311-327.

45 P. A. Hatherly, D. M. Smith and R. P. Tuckett, Z. Phys. Chem., 1996, 195, 97-136.

46 I. R. Lambert, S. M. Mason, R. P. Tuckett and A. Hopkirk, J. Chem. Phys., 1988, 89, 2675.

47 I. R. Lambert, S. M. Mason, R. P. Tuckett and A. Hopkirk, J. Chem. Phys., 1988, 89, 2683.

48 A. G. Sage, T. A. A. Oliver, R. N. Dixon and M. N. R. Ashfold, Mol. Phys., 2010, 108, 945-955.

49 T. Baer, S. H. Walker, N. S. Shuman and A. Bodi, J. Phys. Chem. A, 2012, 116, 2833-2844.

50 E. M. Russell, E. Cudjoe, M. E. Mastromatteo, J. P. Kercher, B. Sztáray and A. Bodi, J. Phys. Chem. A, 2013, 117, 4556-4563. 